\section{Seleção de prioridades para programas de saúde, nutrição e alimentação: um exemplo no estado da Bahia}

\section{Selection of priorities for health and nutrition programs: an example in the state of Bahia}

Leonor Maria Pacheco Santos 1

Manuela da Cruz Gouveia 2

Davi Felix Martins Junior 3

Sandra Maria Chaves dos Santos 4 Maurício Lima Barreto 5

1 Departamento de Nutrição da Universidade Federal de Pernambuco

Organização Panamericana da Saúde, Gerência do Projeto de Micronutrientes Setor Embaixadas Norte, Lote 19, Brasília, DF, Brasil, CEP 70.353-090, leonor@bra.ops-oms.org 2,4 Escola de Nutrição da Universidade Federal da Bahia

3 Departamento de Saúde da Universidade Estadual de Feira de Santana

5 Instituto de Saúde Coletiva da Universidade Federal da Bahia

\section{Abstract}

Objectives: between 1996 and 1998 three public policies in the area of maternal and child health were implemented: Program to Reduce Infant Mortality (PRIM), Community Solidarity Program (CSP) and Incentive to Combat Nutritional Deficiencies (ICND). The objective of this study was to compare the methodology used to select priorities and to choose municipalities for these three public policies.

Methods: the indexes and statistical indicators, as well as the databases from which they originated, the independent and dependent variables and statistical models employed were analyzed, based on the description available in official publications. The state of Bahia was chosen as an example, and the construction of the cartographic database was performed directly on the screen, with the software Epi-Map.

Results: one could observe that different parameters and distinct databases were used to construct each one of the indexes analyzed. As a final result, there was an agreement in only 28 of the 111 municipalities chosen by PRIM, the 96 selected by CSP and the 100 considered priorities by the ICND.

Conclusions: in the case of these three social programs, implemented almost simultaneously in the state of Bahia, involved considerable investments of funds, focusing and targeting, which are key elements for the optimization of public spending in social policies, occurred in only less than 28\% of the cases.

Key words Health priorities, Nutrition Programmes, Nutrition, Infant Mortality, Nutrition Programmes and Policies.

\section{Resumo}

Objetivos: entre 1996 e 1998 foram implantadas três políticas públicas na área de saúde materno-infantil: Programa de Redução da Mortalidade Infantil (PRMI), Programa Comunidade Solidária (PCS) e Incentivo de Combate às Carências Nutricionais (ICCN). Este estudo teve como objetivo comparar a metodologia empregada para selecionar os municípios prioritários nestas três políticas públicas.

Métodos: os índices e indicadores estatísticos, bem como as bases de dados que deram origem aos mesmos, as suas variáveis independentes e dependentes e a modelagem estatística, foram analisados a partir de sua descrição nas publicações oficiais. Foi usado como exemplo o estado da Bahia e a construção da base cartográfica ocorreu pela digitalização do contorno dos municípios, diretamente na tela do programa Epi-Map.

Resultados: pode-se observar que parâmetros distintos e diferentes bases de dados foram empregados para a construção dos três diferentes índices analisados. Como resultado final dentre os 111 municípios selecionados pelo PRMI, os 96 escolhidos pelo PCS e os 100 considerados prioritários dentro do ICCN, verificou-se que houve superposição em apenas 28.

Conclusões: no caso destas três políticas públicas, implantadas quase simultaneamente no estado da Bahia, envolvendo um volume considerável de recursos, em menos do que $28 \%$ dos casos ocorreu o que se pode chamar de focalização e convergência, elementos considerados essenciais para a otimização do investimento dos recursos públicos em políticas sociais. Palavras-chave Prioridades em Saúde, Programas de Nutrição, Nutrição, Mortalidade Infantil, Programas e Políticas de Nutrição e Alimentação. 


\section{Introdução}

Os países em desenvolvimento, incluindo o Brasil, destinam parcelas significativas de recursos aos programas sociais. ${ }^{1}$ Considerando-se a situação econômica e social desses países, os recursos dirigidos aos grupos excluídos representam tanto uma tentativa de superar a situação considerada insatisfatória, como o não-investimento em outras áreas, igualmente prioritárias. Desta forma, estas políticas e programas devem ser capazes de satisfazer duas condições básicas: corrigir a situação-problema e fazê-lo com o maior benefício possível, tendo em vista os limitados recursos disponíveis.

As discussões sobre a questão da segurança alimentar foram introduzidas na Europa a partir da I Guerra Mundial, ligadas à preocupação com a segurança nacional, traduzindo-se pela necessidade de cada país produzir sua própria alimentação, de forma a não se tornar vulnerável a cercos, embargos ou boicotes de motivação política ou militar. Até a década de 70, a segurança alimentar foi compreendida muito mais como uma política de armazenamento estratégico, de oferta segura e adequada de alimentos, do que analisada, mais propriamente, sob a ótica dos direitos humanos. O enfoque principal era o alimento e não o ser humano. ${ }^{2}$

Contudo essa visão se modifica a partir das recomendações da oitava seção do Comitê Internacional de Segurança Alimentar, de 1987. Conforme destacado por Galeazzi ${ }^{3}$, desde então assume-se que a discussão da segurança alimentar implica na garantia do acesso físico e econômico de todos, em todos os momentos, aos alimentos básicos, de acordo com suas necessidades, dependente, portanto, de intervenções no âmbito da produção e da distribuição.

As primeiras referências à segurança alimentar no Brasil ocorreram em meados da década de 80, tendo sido elaborado no Ministério da Agricultura um documento intitulado "Política Nacional de Segurança Alimentar".4 Tal proposta, no entanto, não chegou a ser implementada. $\mathrm{O}$ assunto foi retomado em 1987, quando da realização da $1^{\text {a }}$ Conferência Nacional de Alimentação e Nutrição, um desdobramento temático da $8^{a}$ Conferência Nacional de Saúde. A alimentação foi então defendida como um direito e propôs-se a criação de um Conselho Nacional de Segurança Alimentar (CONSEA) e de um Sistema de Segurança Alimentar. A implantação dessas duas propostas era considerada necessária para garantir o acesso a uma alimentação adequada e segura, devendo o direito à alimentação ser garantido em termos constitucionais e conquistado por meio de múltiplas políticas.
Contudo, a Constituição de 1988,5 considerada como avançada no campo dos direitos sociais, não consagrou um capítulo específico ao direito à alimentação. Esta aparece no texto constitucional vinculada ao salário mínimo, como uma das necessidades básicas a serem cobertas por este salário; caberia ao Sistema Único de Saúde (SUS) apenas a fiscalização e inspeção de alimentos.

No início dos anos 90 ganha destaque um velho dilema nacional: a persistência da pobreza numa magnitude surpreendente e com ela muitos dos seus corolários, entre os quais a fome. 6 Alguns autores consideravam que, no início dos anos 90, a permanência de situações de pobreza e fome denunciavam décadas de má aplicação dos recursos. ${ }^{1}$ Em paralelo surgiram denúncias, cada vez mais intensas e comprovadas, de desvio de verbas públicas desses programas. 7 Nesta época houve um brusco esvaziamento dos programas sociais, a ponto do relatório do Banco Mundial, que analisou o desempenho das políticas dirigidas aos problemas nutricionais no Brasil, revelar que durante certos períodos houve praticamente um colapso de todos os programas de alimentação e nutrição. 8

Em 1993 o Movimento pela Ética na Política, em parceria com o Instituto Brasileiro de Análises Sociais e Econômicas (IBASE), Ordem dos Advogados do Brasil (OAB), Conferência Nacional dos Bispos Brasileiros (CNBB), Sociedade Brasileira para o Progresso da Ciência (SBPC), Central Única dos Trabalhadores (CUT), outras entidades e diversas personalidades da sociedade civil, lança, com ampla repercussão, a "Ação da Cidadania contra a Fome, a Miséria e pela Vida". 9 Como respostas governamentais, a presidência da República cria o Conselho Nacional de Segurança Alimentar, (CONSEA) através do Decreto n. ${ }^{\circ} 807$ de 24 de abril de 1993 e cria, neste mesmo ano, o Programa de Atenção aos Desnutridos e Gestantes em Risco Nutricional - Programa Leite é Saúde (PLS). A implantação deste último ocorreu a partir de 1995 e sua estratégia básica era a suplementação alimentar com leite e óleo de soja, para crianças e gestantes de baixo peso. 10

No relatório final da $1^{\text {a }}$ Conferência Nacional de Segurança Alimentar, organizada e conduzida pelo CONSEA em 1994, consta que: "... haverá segurança alimentar quando todos os brasileiros tiverem permanentemente acesso em quantidade e qualidade aos alimentos requeridos para a saudável reprodução do organismo humano e para uma existência digna" (CONSEA ...; 1994: 6).11

Em termos internacionais, a Reunião de Cúpula Mundial sobre Alimentação, organizada pela Food and Agriculture Organization (FAO) em novembro 
de 1996, produziu a Carta de Roma sobre a Segurança Alimentar Mundial, assinada por chefes de Estado de países membros das Nações Unidas, inclusive o Brasil. O documento "... reafirma o direito de todos ao acesso a alimentos sadios e nutritivos, em consonância com o direito a uma alimentação apropriada e com o direito fundamental das pessoas de não passar fome." Considera ainda que "... existe Segurança alimentar quando todas as pessoas têm, em todo momento, acesso físico e econômico a alimentos suficientes, inócuos e nutritivos, para satisfazer suas necessidades alimentares e suas preferências quanto a alimentos, a fim de levar uma vida ativa e sadia."(FAO; 1996). ${ }^{12}$

Contudo, cabe registrar o paradoxo entre os compromissos internacionais firmados pelo governo brasileiro nesta área e a descontinuidade das estruturas nacionais destinadas ao enfrentamento do problema, evidenciando a distância existente entre o discurso e a prática. Desde 1987 se propunha a criação de um Conselho Nacional de Segurança Alimentar e de um Sistema de Segurança Alimentar no Brasil, resoluções da $1^{a}$ Conferência Nacional de Alimentação e Nutrição.11 Finalmente em 1993 foi criado o CONSEA, como órgão de aconselhamento da Presidência da República, com ampla representação de órgãos do governo e da sociedade civil organizada e presidido por um membro do Clero. Surpreendentemente, o mesmo foi extinto por decreto em fins de 1994, num estilo autoritário e antidemocrático, não condizente com o discurso governamental da época; uma nova política social, denominada Programa Comunidade Solidária (PCS), foi criada com a proposta de absorver, entre outros, os componentes de segurança alimentar do CONSEA. 13

Em 1995, devido à persistência de elevados níveis de mortalidade infantil nos estados do Nordeste e, em particular, o seu recrudescimento nos cinco anos precedentes, o Ministério da Saúde criou o Programa de Redução da Mortalidade Infantil (PRMI), em parceria com as Secretarias Estaduais de Saúde, destacando como meta prioritária, a redução dos coeficientes de mortalidade na infância, através de uma abordagem intersetorial. 14

No tocante à suplementação alimentar com leite e óleo de soja, iniciada na década de 90 pelo Programa Leite é Saúde, 10 o Incentivo ao Combate às Carências Nutricionais (ICCN), criado em março de 1998, deu continuidade a esta linha de ação, entre as políticas públicas implementadas pela Área Técnica de Alimentação e Nutrição, recém criada no Ministério da Saúde.

O presente estudo teve como objetivo analisar a seleção dos municípios prioritários contemplados nos três programas sociais listados abaixo, que, seguramente, representaram os investimentos mais importantes voltados à população materno-infantil na década dos 90:

Programa de Redução da Mortalidade na Infância (PRMI);

Programa da Comunidade Solidária (PCS);

Incentivo ao Combate às Carências Nutricionais (ICCN).

\section{Aspectos metodológicos}

Cumpre inicialmente descrever de modo sucinto os três programas sociais em pauta, com o propósito de explicitar seus objetivos, modo de atuação e sobretudo a metodologia de seleção dos municípios prioritários.

\section{Programa de Redução da Mortalidade na Infância}

Em março de 1995, o Ministério da Saúde, em reunião realizada no estado de Pernambuco, solicitou dos nove Secretários da Saúde do Nordeste um projeto para que fossem trabalhadas todas as dificuldades relacionadas com o problema da mortalidade infantil em cada estado.

Os Secretários, presentes à reunião do planejamento conjunto dos projetos de redução da mortalidade na infância, lançaram naquela ocasião a "Carta de Olinda", documento através do qual se comprometeram a dar apoio absoluto a esta proposta do Ministério da Saúde.14

O Ministério da Saúde firmou compromisso de acompanhar a implantação e a implementação do projeto, além de fornecer contrapartida em medicamentos e em insumos básicos, num processo de acompanhamento, que deveria ser feito com os demais setores de desenvolvimento, como os de saneamento, agricultura, educação, etc. Como plano de ação, o PRMI é coordenado pelas Secretarias da Saúde, em parceria com outras Secretarias como Justiça, Trabalho e Ação Social, Saneamento, Educação, Agricultura, Planejamento, com o Tribunal de Justiça e com instituições como Fundação Nacional de Saúde e Pastoral da Criança, além da colaboração técnica do Fundo das Nações Unidas para a Infância (UNICEF).

A estratégia inicial do PRMI na Bahia foi a seleção dos 111 municípios de risco e a formação do Grupo de Trabalho Inter-Setorial Estadual (GTI), integrado por representantes das diferentes Secretarias e Instituições. Posteriormente houve a formação de 
GTIs regionais e municipais, na medida em que ocorreu a implantação e implementação do PRMI nas regiões e municípios dos estados. As estratégias e ações foram subdivididas em 4 áreas temáticas: a) saúde e saneamento; b) assistência social e judiciária; c) educação e d) produção; elaboradas conjuntamente, através do trabalho inter-setorial.

O PRMI tem como objetivo geral melhorar os níveis de saúde infantil nos municípios brasileiros, dando prioridade aos "municípios de risco" nos primeiros cinco anos de implantação e incluindo os demais no período subseqüente. A seleção dos municípios prioritários baseou-se fundamentalmente no Índice de Condições de Sobrevivência (ICS) calculado e publicado pela Instituto de Geografia e Estatística (IBGE) e UNICEF15 a partir de indicadores sociais e econômicos. O programa PRMI tem as seguintes metas explicitadas e quantificadas:

Reduzir em $10 \%$ ao ano a taxa de mortalidade materna;

Reduzir em $15 \%$ ao ano a taxa de mortalidade infantil;

Reduzir em $15 \%$ ao ano a taxa de mortalidade em menores de 5 anos;

Reduzir em $10 \%$ ao ano a taxa de desnutrição em menores de 5 anos.

\section{Programa Comunidade Solidária}

Em 1995, o recém-empossado governo federal extinguiu o aparato estatal de assistência social preexistente no Brasil, avaliado, por administradores, políticos, estudiosos e beneficiários, como ineficiente e permeado de práticas clientelistas, e criou o Programa Comunidade Solidária através do Decreto $\mathrm{n}^{\circ}$ 1.366, 12 de Janeiro de 1995.

O PCS define como seu objetivo mais geral "o atendimento da parcela da população que não dispõe de meios para prover suas necessidades básicas $e$, em especial, o combate à fome e à pobreza". Associado a este objetivo, a política do programa adota ainda como proposições a descentralização, a parceria, a solidariedade e a ação convergente e integrada. Além disso, assume a focalização como uma estratégia fundamental, de forma a atender os municípios mais pobres do país e nestes a parcela de sua população mais vulnerável do ponto de vista social. Visa também maior participação da sociedade na gestão dos programas integrados à sua agenda, de forma a melhorar a eficiência e eficácia das ações, incentivar o controle social e superar práticas políticas tais como o clientelismo, estatismo, fisiologismo e corporativismo. 16,17

O PCS traduz-se na prática em um grande con- junto de programas e projetos setoriais, não necessariamente novos, que passam a integrar uma política pública de combate à pobreza e à fome, descritos a seguir: Programa de Combate à Desnutrição Materno-Infantil, Programa de Saneamento, Programa Nacional de Imunizações, Programa de Agentes Comunitários de Saúde, Programa Nacional de Alimentação Escolar, Programa de Distribuição de Alimentos, Programa Cesta Saúde do Escolar, Programa de Assistência Integral à Saúde do Escolar, Programa Nacional de Transporte Escolar, Programa de Educação Infantil, Programa de Ação Social em Saneamento, Programa Habitar-Brasil, Programa Nacional de Fortalecimento da Agricultura Familiar, Programa de Geração de Emprego e Renda e Plano Nacional de Educação Profissional.13

A seleção dos municípios incluídos no PCS, que deveria privilegiar os mais pobres de cada estado, foi realizada pela Secretaria Executiva da Comunidade Solidária em consulta com instâncias a nível estadual (no caso da Bahia, a Secretaria de Planejamento). Como parâmetros para a determinação da pobreza municipal foram combinados dois grupos de indicadores:

\section{Indicadores de Indigência - Mapa da fome II / IPEA}

Esse indicador classifica os municípios de acordo com a proporção de famílias indigentes, cuja renda é insuficiente para sua subsistência. 18

\section{Índice de Condições de Sobrevivência}

Ordena municípios com base nas condições de sobrevivência de crianças de 0 a 6 anos de idade, a partir de indicadores sociais e econômicos; trata-se do mesmo indicador empregado pelo PRMI. ${ }^{15}$

Os municípios que apresentavam índices mais insatisfatórios foram incluídos na relação, sempre que a soma de famílias indigentes alcançasse o limite estabelecido pelo IPEA, com base nos dados do IBGE. 17

Em 1995 o número de municípios prioritários selecionados pelo PCS foi de 302 em todo o Brasil, com recursos de $\mathrm{R} \$ 983.200,00$ aplicados pelos programas da agenda básica. Já em 1996 este número se elevou para 1.111 municípios e os recursos para $\mathrm{R} \$ 1.402 .000,00$. No estado da Bahia foram 96 os municípios selecionados e incluídos no PCS em 1996. A partir de então (de 1997 a 2000) o número de municípios contemplados pelo Programa estabilizou-se em 1.368. 


\section{Incentivo ao Combate às Carências Nutricionais}

A pressão social gerada pela Ação da Cidadania Contra a Fome, a Miséria e pela Vida, conforme citado anteriormente, ocasionou como resposta governamental, a criação do CONSEA e a implantação de um novo programa de suplementação alimentar, o Programa Leite é Saúde (PLS), com vistas a contribuir tanto para a formulação e coordenação de uma Política Nacional de Saúde, como para a municipalização das ações de saúde, na direção da consolidação do Sistema Único de Saúde ${ }^{10}$. O Programa de Atenção aos Desnutridos e Gestantes em Risco Nutricional - Leite é Saúde - foi criado através da Portaria do Ministério da Saúde $\mathrm{n}^{\circ} 799$, de julho de 1993, visando a distribuição de leite em pó (ou fluido) e de óleo de soja a crianças e gestantes de baixo peso e aos seus contatos (irmãos das crianças de baixo peso, na faixa etária de 0 a cinco anos de idade). O repasse de recursos para que os municípios adquirissem os alimentos era feito aos através de convênios individuais, firmados com o Instituto Nacional de Alimentação e Nutrição (INAN).

Posteriormente, com a extinção do INAN, o Programa Leite é Saúde foi substituído pelo Incentivo de Combate às Carências Nutricionais (ICCN), criado através da Portaria n 2.409 de março de 1998, no qual foi instituido o mecanismo de repasse de recursos fundo a fundo (do Fundo Nacional de Saúde ao Fundo Municipal de Saúde). O ICCN tem como público alvo crianças de baixo peso (abaixo do Percentil 10 do "cartão da criança") na faixa etária de 6-23 meses e gestantes com baixo peso em relação à altura, de acordo com o "cartão da gestante". O ICCN prevê a distribuição de $1.000 \mathrm{ml}$ de leite/dia (ou $3,6 \mathrm{~kg} / \mathrm{mês}$ ) e uma lata de óleo de soja/mês por beneficiário. Os principais pré-requisitos para a implantação do ICCN no município são: a) aprovação do Plano de Combate às Carências Nutricionais pelo Conselho Municipal de Saúde; b) Sistema de Vigilância Alimentar e Nutricional implantado e c) cumprimento das normas do SUS para municipalização dos serviços de saúde. Ao Ministério da Saúde cabe a coordenação nacional das ações previstas, inclusive a autorização para repasse de recursos fundo a fundo. Os municípios procedem a aquisição dos alimentos, seu armazenamento, distribuição e o acompanhamento dos beneficiários. No âmbito estadual a responsabilidade centra-se na análise prévia dos Planos Municipais do ICCN, prevendo-se também a supervisão da sua execução.

Através do ICCN o Ministério da Saúde repassa aos municípios $\mathrm{R} \$ 180,00$ por criança desnutrida/ano. O número estimado de desnutridos por mu- nicípio consta da Portaria no 2.409 de março de 1998 e foi baseado na estimativa de ocorrência de desnutrição calculada pela equipe do Núcleo de Pesquisa Epidemiológicas em Nutrição e Saúde da Universidade de São Paulo (NUPENS/USP) para os municípios brasileiros através de indicadores sociais e econômicos. ${ }^{19}$ Em 1998 foram 278 os municípios baianos habilitados pelo ICCN, recebendo um montante de recursos de $\mathrm{R} \$ 7.666 .897,50$.

\section{Desenho do estudo}

Foram analisados e comparados os índices, os indicadores e a metodologia empregados para selecionar os municípios prioritários nas três políticas públicas descritas acima, que seguramente representaram aquelas de maior peso e com maior inversão de recursos na área de saúde materno-infantil dos anos 90. Os índices e indicadores estatísticos, bem como as bases de dados que deram origem aos mesmos, as suas variáveis independentes e dependentes e a modelagem estatística, foram analisados a partir de sua descrição nas publicações oficiais que nortearam o delineamento dos programas supracitados.14,15,18,19

Os resultados finais, em termos da comparação de municípios selecionados, usaram como exemplo o estado da Bahia. Este estado é um dos nove que compõem a região Nordeste do Brasil, com uma área de $559.951 \mathrm{~km}^{2}$ (terras emersas), correspondendo a cerca de 7\% do território nacional. Esta unidade espacial de referência para a pesquisa estava politicamente dividida em 336 municípios em 1980, evoluindo para 415 municípios em 1991, divisão esta que vigorou até 1999. No ano de 2000 foram criados mais dois municípios, perfazendo um total de 417 municípios. Segundo o último censo realizado em 1991, o Estado da Bahia abrigava 11.867.991 habitantes, evoluindo para 12.541.675 habitantes em 1996, ano da Contagem Populacional, ocupando a quarta posição em termos de população absoluta no conjunto da federação. ${ }^{20}$ Em relação a 1991, ocorreu um aumento populacional superior a seiscentas e setenta mil pessoas.

Do ponto de vista econômico, o estado da Bahia é a quinta economia da federação. A área incluída no polígono das secas na Bahia corresponde a 393.000 $\mathrm{km}^{2}$, ou seja, mais que $2 / 3$ da área total do estado. $\mathrm{O}$ processo de construção da base cartográfica ocorreu pela digitalização do contorno dos municípios diretamente na tela do Epi-Map, programa de domínio público distribuído pela Organização Mundial da Saúde e pelo Center for Disease Control (CDC), em Atlanta, Estados Unidos. 


\section{Resultados e Discussão}

Os parâmetros empregados para a construção dos três diferentes índices analisados, tais como a base de dados, as variáveis independentes e dependentes, a modelagem estatística e os pontos de corte estão apresentados, sumariamente, na Quadro 1.

Quadro 1

Características dos índices sociais e econômicos empregados no planejamento de políticas de saúde e nutrição.

\begin{tabular}{|c|c|c|c|c|c|}
\hline Índice & Base de dados & $\begin{array}{l}\text { Modelo } \\
\text { Estatístico }\end{array}$ & $\begin{array}{l}\text { Variável } \\
\text { Dependente }\end{array}$ & Variáveis Independentes & Pontos de Corte \\
\hline $\begin{array}{l}\text { Índice de Condições de. } \\
\text { Sobrevivência } 15\end{array}$ & Censo 1991 - IBGE & Regressão múltipla & Mortalidade infantil & $\begin{array}{l}\text { Chefe com renda }<1 \mathrm{SM}^{*} \\
\text { Chefe } \mathrm{H}<1 \text { ano de estudo } \\
\text { Chefe } \mathrm{M}<1 \text { ano de estudo } \\
\text { Abast. água inadequado }\end{array}$ & $\begin{array}{l}\text { 1,00-0,50 Baixa } \\
\text { 0,49-0,30 Intermediária } \\
\text { 0,29-0,00 Boa condição } \\
\text { de sobrevivência } \\
\text { infantil }\end{array}$ \\
\hline Mapa da Fome 18 & $\begin{array}{l}\text { PNAD } 1990 \text { - IBGE } \\
\text { Censo 1980- IBGE } \\
\text { INPC alimentação- Recife } \\
\text { Censo } 1991 \text { - IBGE (dados } \\
\text { preliminares) }\end{array}$ & $\begin{array}{l}\text { Não foi informado } \\
\text { (PNAD- ajuste pela Função } \\
\text { de Pareto) }\end{array}$ & Não foi informado & Não foi informado & $\begin{array}{l}\text { Proporção de famílias } \\
\text { auferindo rendimentos } \\
\text { de até } 2 \mathrm{SM}\end{array}$ \\
\hline $\begin{array}{l}\text { Risco de Desnutrição } \\
\text { Infanti|19 }\end{array}$ & $\begin{array}{l}\text { PNSN } 1989 \text { - INAN/IBGE } \\
\text { Censo } 1991 \text { - IBGE }\end{array}$ & Regressão logística & Altura para idade $<-2 d p$ & $\begin{array}{l}\text { Alfabetização da } \\
\text { mãeRenda do chefe } \\
\text { família Saneamento } \\
\text { ( } \text { modelos para as zonas } \\
\text { urbana e rural) }\end{array}$ & $\begin{array}{l}\text { Prevalência de } \\
\text { desnutrição calculada } \\
\text { para cada um dos } \\
\text { municípios brasileiros }\end{array}$ \\
\hline
\end{tabular}

SM = Salário Mínimo

$\mathrm{H}=\mathrm{Homem}$

$\mathrm{M}=$ Mulher

No caso do Mapa da Fome as publicações oficiais não informam sobre a modelagem estatística, nem sobre as variáveis dependentes e independentes; este índice também pode ter ficado prejudicado pelo fato de basear-se, em parte, nos dados censitários antigos (censo 1980).

A análise da distribuição espacial dos municípios prioritários nestes três programas está apresentada nas Figuras de 1 a 4 . Os 111 municípios contemplados pelo Programa de Redução da Mortalidade na Infância foram selecionados pelo Ministério da Saúde e Secretaria Estadual de Saúde da Bahia, baseados, em princípio, no Índice de Condições de Sobrevivência Infantil15 e estão ilustrados na Figura 1. Os municípios considerados de risco se encontraram distribuídos por todas as regiões da Bahia, incluindo o extremo sul do estado e a região oeste (do além-São Francisco). Chamou atenção o fato de existirem faixas na região central e na região norte do estado onde praticamente nenhum município foi incluído, apesar de se constituírem em regiões muito áridas do sertão da Bahia, onde freqüentemente são implantados programas de frentes de emergência. 
Distribuição espacial dos 111 municípios prioritários selecionados para participar no Programa de Redução da Mortalidade na Infância (PRMI). Bahia, 1996.

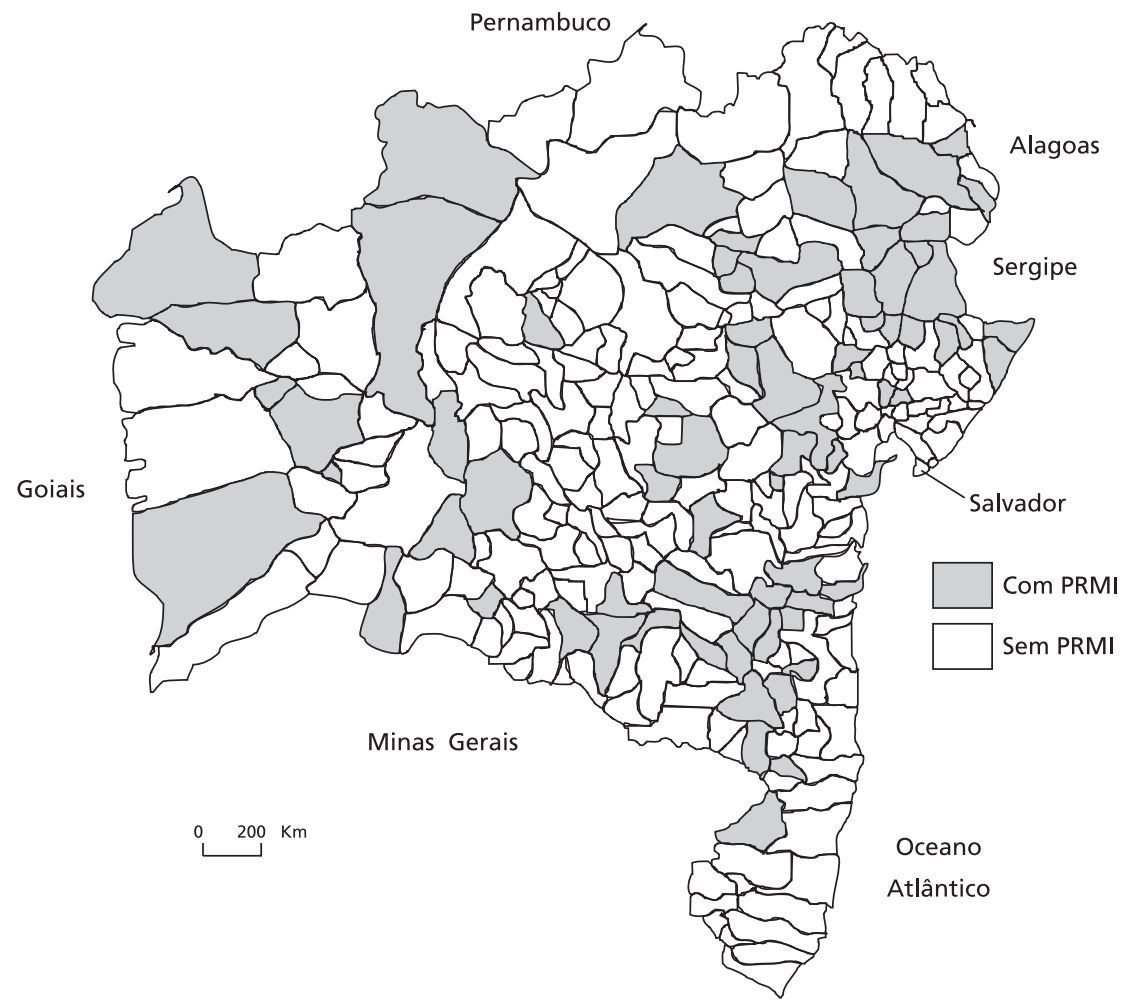

A coordenação do Programa Comunidade Solidária, em conjunto com a Secretaria de Planejamento do Estado da Bahia, elegeu 96 municípios como prioritários para a implantação do PCS na Bahia (Figura 2). Esta seleção baseou-se fundamentalmente nos resultados do Mapa da Fome-II 18 e no Índice de Condições de Sobrevivência. 15 Pode-se verificar que nenhum dos municípios do Extremo
Sul foi contemplado, ao passo que na região central do estado, onde o PRMI apresentou uma cobertura muito baixa, o PCS incluiu 14 municípios. No estado como um todo, houve uma baixa concordância entre os municípios do PRMI e do PCS, pois ocorreu uma superposição entre os dois programas apenas no caso de 50 municípios (menos do que $50 \%$ de concordância). 
Distribuição espacial dos 96 municípios prioritários selecionados para participar no Programa Comunidade Solidária (PCS). Bahia, 1996

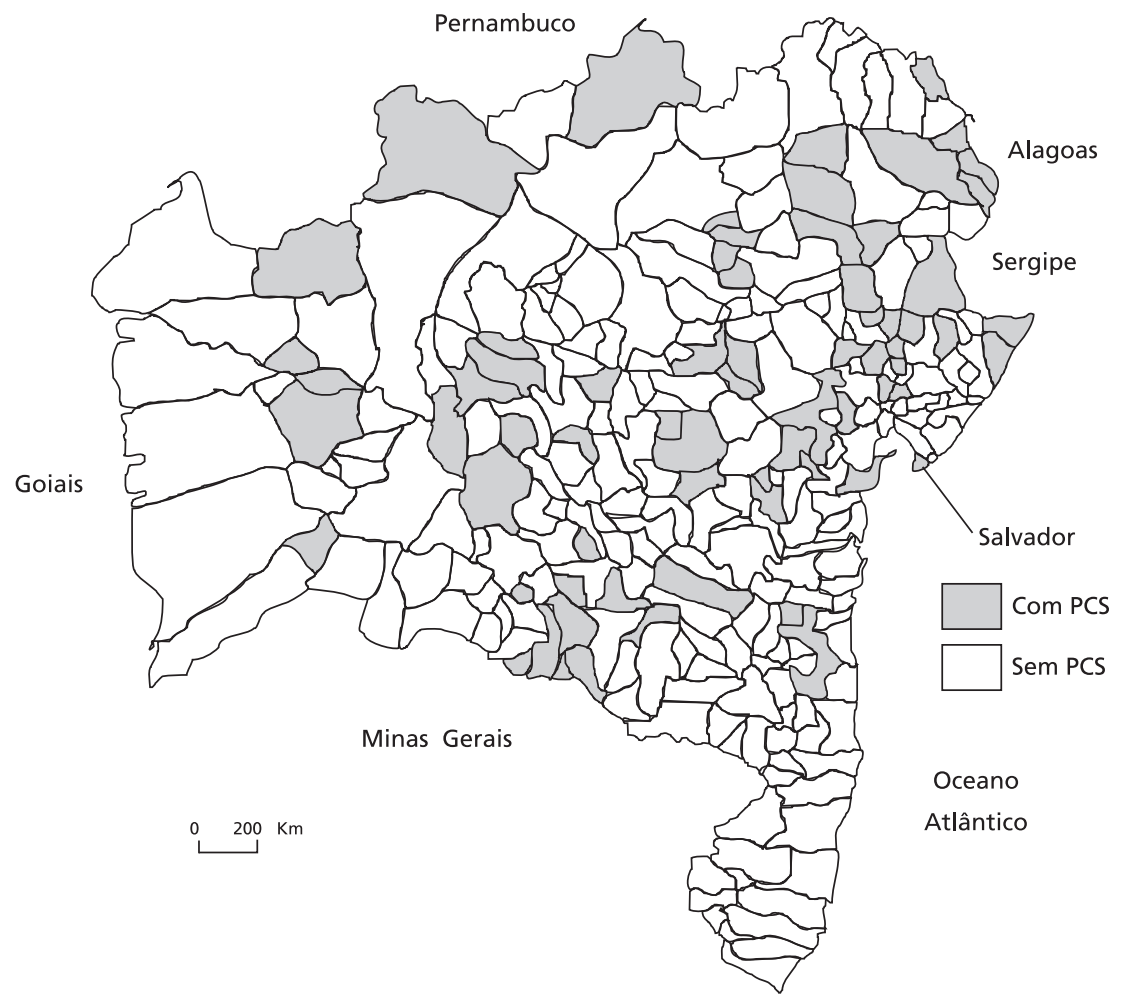

Já em relação ao Incentivo ao Combate às Carências Nutricionais, a sua cobertura prevista é de $100 \%$, ou seja, em princípio todos os 415 municípios baianos eram elegíveis. Contudo o programa elencou prioridades, na medida em que o volume de recursos é determinado de acordo com a maior ou menor prevalência de desnutrição no município, calculada pelo Risco de Ocorrência de Desnutrição Infantil. ${ }^{19}$ Assim sendo, para fins desta análise comparativa, foram incluídos no mapa da Figura 3, os
100 municípios prioritários na Bahia, ou seja, aqueles com maior prevalência de desnutrição e que receberam, proporcionalmente, as maiores verbas do ICCN (o que correspondeu aos municípios com prevalência de desnutrição superior a $31,87 \%$ ). Na comparação entre os municípios selecionados pelo PRMI e os considerados prioritários pelo ICCN, houve uma concordância ainda menor, pois apenas 34 deles coincidiram. 
Distribuição espacial dos 100 municípios prioritários para o Incentivo ao Combate às Carência Nutricionais (ICCN), Bahia, 1998.

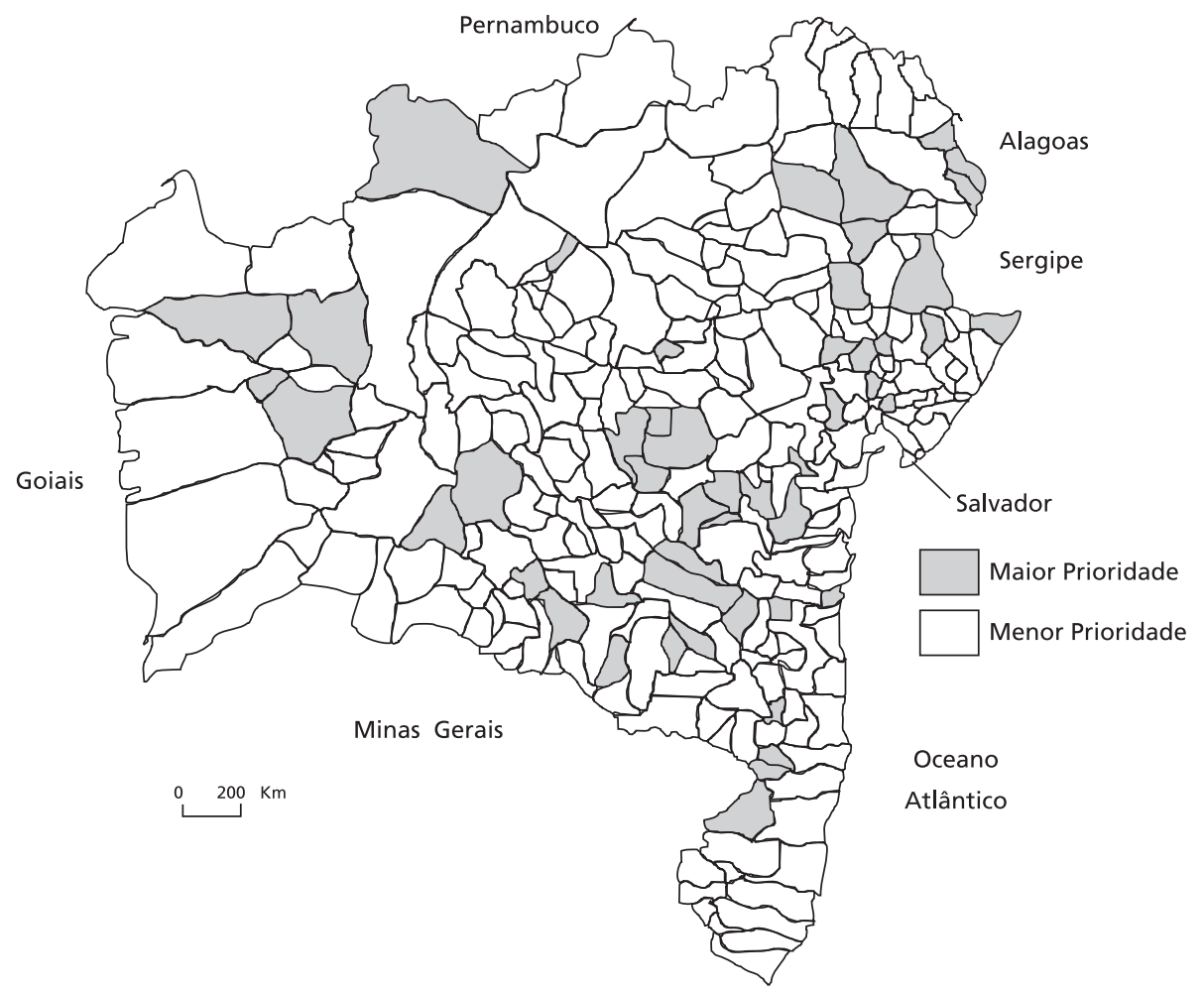

A Figura 4 condensa os achados ilustrados pelos três mapas anteriores para evidenciar os municípios onde houve concordância no caso dos três programas, de dois deles ou de apenas um. Pode-se verificar que houve superposição completa em apenas 28 municípios baianos. Ou seja, nestas três políticas públicas, implantadas quase que simultaneamente no estado da Bahia, em apenas 28 casos houve o que se pode chamar de focalização e convergência, elementos considerados essenciais para uma otimização do investimento de recursos públicos. 
Distribuição espacial dos municípios selecionados como prioritários em três diferentes programas sociais no estado da Bahia, 1996-1998

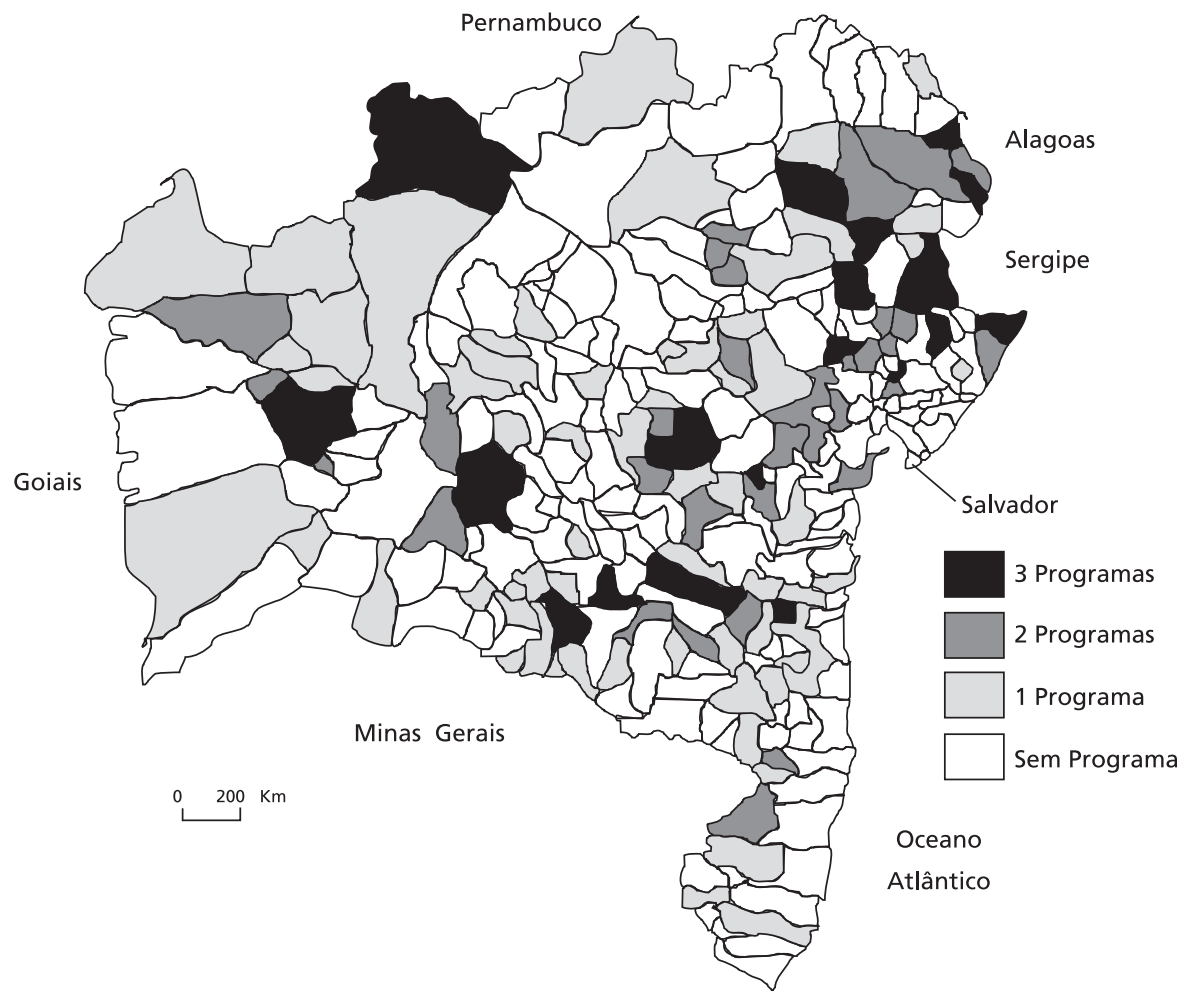

\section{Conclusões e Recomendações}

Os resultados obtidos demonstram que o uso de diferentes indicadores sociais e econômicos está longe de produzir resultados concordantes na definição de prioridades para implantação de programas sociais, e em especial de programas de saúde, nutrição e alimentação. Os achados apontam para a importância da realização de estudos direcionados à validação dos diversos indicadores possíveis de serem construídos com as bases de dados existentes no país, antes que os mesmos sejam utilizados por gestores de políticas e programas, nos processos de tomada de decisão, de definição de prioridades e de alocação de recursos.

Neste estudo fez-se uma análise dos resultados obtidos pela aplicação de diferentes critérios para definição de prioridades, tendo em vista a seleção de municípios baianos contemplados por três progra- mas voltados para problemas que afetam a população materno-infantil. Observou-se que os avanços que podem ser identificados nos diagnósticos e nas proposições não se traduziram, até o presente, na adoção de critérios capazes de viabilizar a focalização convergente e integrada. Como visto, apenas em 28 dos 415 municípios baianos, cerca de $7 \%$ dos casos, os três programas convergiram para um mesmo território.

Além desse aspecto, importa considerar que uma vez selecionado o território, cada um dos programas adota determinados critérios para selecionar sua clientela alvo. Isto é particularmente real para o Programa Comunidade Solidária, o qual se configura como uma cesta de programas setoriais. Assim, ainda que convergindo para um determinado município, os programas tendem a não convergir para um mesmo grupo populacional, promovendo nova fragmentação. 
Com esses resultados, conclui-se que a adoção de diferentes indicadores sociais e econômicos por programas que pretendem intervir de forma articulada, não vem contribuindo para o alcance do objetivo de maximizar a aplicação focalizada e convergente dos escassos recursos, incorrendo na perseverança da pulverização e de suas conseqüências sobre a falta de efetividade e eficácia dos programas.

É reconhecido que a mensuração da ocorrência de problemas de natureza social é complexa, o que impõe limites à discussões sobre a adequação de um ou outro critério para classificação de regiões, populações e indivíduos, enquanto potenciais beneficiários de programas sociais. No entanto, o planejamento da ação governamental exige a adoção de critérios, os quais traduzem algum tipo de trade off.

Nessa perspectiva, sugere-se que a discussão sobre critérios para seleção de prioridades caminhe na direção de uma padronização que seja sensível o bastante para captar o fenômeno social e para subsidiar decisões menos fragmentadoras do tecido social e menos pulverizadoras dos recursos disponíveis. Recomenda-se ainda a realização de mais estudos direcionados à validação dos diversos indicadores possíveis de serem construídos com as bases de dados existentes no país, antes que os mesmos sejam adotados por gestores de políticas e programas no processo de tomada de decisões sobre prioridades para alocação dos recursos disponíveis.

\section{Referências}

1. Draibe S. As políticas sociais brasileiras: diagnóstico e perspectivas. In: IPEA. Instituto de Pesquisa Econômica Aplicada. Prioridades e perspectivas das políticas públicas para a década de 90. Brasília, DF: IPEA; 1989. p. 166

2. IPEA. Instituto de Pesquisa Econômica Aplicada. Mapa da fome; subsídios à formulação de uma política de segurança alimentar. Brasília, DF: IPEA; 1993. (Documento de política, 14)

3. Galeazzi MAM. A segurança alimentar e os problemas estruturais de acesso. In Galeazzi MAM. Segurança alimentar e cidadania: a contribuição das universidades paulistas. Campinas: Mercado de Letras; 1996. p.133-56

4. IPEA. Instituto de Pesquisa Econômica Aplicada, Conselho da Comunidade Solidária. O Conselho da Comunidade Solidária e a segurança alimentar e nutricional no Brasil documento-base. Brasília, DF: IPEA; 1996

5. Brasil. Constituição, 1988. Constituição da República Federativa do Brasil. São Paulo: Revista dos Tribunais; 1988.

6. Lavinas L. Acessibilidade alimentar e estabilização econômica no Brasil nos anos 90. Rio de Janeiro: IPEA. Instituto de Pesquisa Econômica Aplicada; 1998.

7. Gonçalves F. Auditoria operacional nos programas de suplementação alimentar do governo federal. Brasília, DF: Tribunal de Contas da União; 1993

8. World Bank. Latin America and Caribbean Regional Office. Brazil: addressing nutritional problems in Brazil: report n $16010-B R$. Washington, DC: The World Bank; 1996.

9. Movimento Ação da Cidadania. Nação cidadania Amazonas 1997; 1(1). Disponível em http://www.internext.com.br/cidadania/histo.htm

10. INAN. Instituto Nacional de Alimentação e Nutrição. Normas operacionais: Programa de Atenção aos Desnutridos e Gestantes em Risco Nutricional. Brasília, DF: INAN; 1993.

11. CONSEA. Conselho Nacional de Segurança alimentar. Relatório final. In: I Conferência Nacional de Segurança Alimentar; 1994 jul 27-30; Brasília, DF, Brasil. Brasília, DF: CONSEA; 1994

2. FAO. Food and Agriculture Organization. Declaración de Roma sobre la seguridad alimentaria mundial y plan de acción de la cumbre mundial sobre la alimentación. Roma: FAO; 1996. Disponível: http://www.fao.org./wfs/final/rd-s.htm. [1999 out 15].

13. Schmitz BAS, Von der Heide ME, Cintra IP, Franceschin SMC, Taddei JÁ, Sigulem DM. Políticas e programas governamentais de alimentação e nutrição no Brasil e sua involução. Cad Nutr 1997; 13: 39-54.

14. Bahia. Secretaria de Saúde. Programa de Redução da Mortalidade na Infância no estado da Bahia. Salvador: Secretaria Estadual de Saúde; 1995.

15. IBGE. Instituto Brasileiro de Geografia e Estatística UNICEF. Fundo das Nações Unidas para a Infância. Municípios brasileiros; crianças e suas condições de sobrevivência. Brasília, DF: IBGE; 1994.

16. Brasil. Presidência. Casa Civil. Manual de orientação às prefeituras municipais. Brasília, DF: Programa Comunidade Solidária; 1997

17. Brasil. Secretaria Executiva. Programa Comunidade Solidária, 1996. Disponível: http://www.planalto.gov.br/SecExec/Criterio.htm

18. Peliano AM. Mapa da fome II. Brasília, DF: IPEA. Instituto de Pesquisa Econômica Aplicada; 1993. (Documento de política, 15 )

19. Benício MH, Monteiro CA. Desnutrição infantil nos municípios brasileiros; risco de ocorrência. São Paulo. Núcleo de Pesquisas Epidemiológicas em Nutrição e Saúde da Universidade de São Paulo (NUPENS); 1998.

20. IBGE. Instituto Brasileiro de Geografia e Estatística. Censo demográfico-Bahia: 1991 (resultados preliminares). Rio de Janeiro: IBGE; 1992. 